\title{
Editorial \\ The quest for the Holy Grail: a disease-modifying osteoarthritis drug
}

Francis Berenbaum ${ }^{1,2}$

\begin{abstract}
1'Department of Rheumatology, APHP Saint-Antoine Hospital, 184 rue du faubourg Saint-Antoine, 75012 Paris, France
2UMR 7079 CNRS, Physiology and Physiopathology Laboratory, University Paris 6, Quai St-Bernard, Paris, 75252 Cedex 5, France
\end{abstract}

Corresponding author: Francis Berenbaum, francis.berenbaum@sat.aphp.fr

Published: 10 December 2007

Arthritis Research \& Therapy 2007, 9:111 (doi:10.1186/ar2335)

This article is online at http://arthritis-research.com/content/9/6/111

(C) 2007 BioMed Central Ltd

See related research by Krzeski et al., http://arthritis-research.com/content/9/5/R109

\begin{abstract}
The unfortunate story of the matrix metalloproteinase inhibitor PG116800, which had no effect on the osteoarthritic process but had unexpected side effects, highlights the following. First, reality does not always match the theory. Second, cell biology data must be interpreted within the context of a specific environment. Third, the specificity of an enzyme inhibitor is always relative. Finally, a critical evaluation of the benefit/risk ratio of a drug must be carefully conducted and checked before and after launch. Well designed post-marketing surveillance is mandatory.
\end{abstract}

The process leading to the discovery of a new drug has completely changed during the past few years. Most therapeutic molecules launched before the 1990s were discovered by unsophisticated screening tests or by chance, without any hypothesis as to their mechanism of action. Studies on mechanism were usually performed after the drug had appeared on the market, sometimes many years after its commercialization. The late 20th century saw the beginning of unparalleled advances in the field of molecular biology that have turned drug discovery upside down. Drug development is now based on basic research findings, such as the effects of over-expression or deletion of genes encoding novel proteins or enzymes in specific in vitro systems. This potential target is then tested in vivo in a translational research process. Clinical trials are then conducted to determine whether the risk/benefit ratio is better than that for existing treatments. This approach is, of course, much more attractive than the previous one because it relies on an hypothesis rather than chance. The development of anti-tumour necrosis factor drugs is a good example of this exciting process.

There should have been a similar success story for the antimatrix metalloproteinase (MMP) drugs in the treatment of osteoarthritis (OA). Basic research revealed that the MMPs, a family of zinc-containing proteinases, are key enzymes in the breakdown of extracellular matrix. In vitro and in vivo experiments confirmed that this was also true for the cartilage matrix. These findings led to the paradigm that destructive arthritis is the result of an imbalance between matrix breakdown and matrix formation in favour of MMP-induced degradation. It was logical, then, that pharmaceutical companies were tempted to seek MMP inhibitors, based on the hypothesis that these compounds would block matrix breakdown.

The report by Spector and coworkers [1] shows that biological systems are sometimes much more complex than predicted. The MMP inhibitor PG-116800 did not modify matrix structure in OA patients. Also, it had unexpected side effects on muscle and skeleton; it limited joint mobility, and caused arthralgia, hand oedema, palmar fibrosis, Dupuytren's contracture and persistent tendon thickness or nodules. This unfortunate story is not the only one in the field of anti-arthritic drugs. Many hoped that bisphosphonates would be diseasemodifying anti-osteoarthritis drugs because many studies demonstrated that the subchondral bone plays a major role in the pathophysiology of OA. However, the phase III pivotal trial to assess the effect of risedronate yielded negative findings [2]. Fortunately, the drug had no serious side effects, and trials of other bone-targeted drugs are ongoing. IL-1 receptor antagonist is another recent example. The receptor antagonist of IL-1 had no clinical effect because it did not counteract IL-1, which is the main cytokine involved in cartilage degradation, when it was injected intra-articularly into OA knees. These unexpected negative results raise several points.

First, reality does not always match the theory. Many biological pathways remain to be discovered, although they may be critical because they interact with a potential drug

$\mathrm{IL}=$ interleukin; $\mathrm{MAPK}=$ mitogen-activated protein kinase; $\mathrm{MMP}=$ matrix metalloproteinase; $\mathrm{OA}=$ osteoarthritis. 
target, making the drug ineffective or producing unexpected side effects. The infliximab trial for the treatment of heart failure is a bizarre example [3]. Although the theory and some experimental data indicated that tumour necrosis factor- $\alpha$ played a major role in the development of heart failure, infliximab not only had no effect but made the disease worse, and so heart failure is now an absolute contraindication for use of this drug.

Second, cell biology data must be interpreted within the context of a specific environment, which could differ dramatically from one person to another. Better interactions between specialties must be encouraged in order to share results on the same pathway but in different contexts. For example, MMPs can be deleterious in some diseases, such as OA, but they may protect the body in others [4]. A systems biology approach could help to limit such surprises [5].

Third, the specificity of an enzyme inhibitor is always relative. It depends on many factors such as cell type, concentration and of course the target itself. A broad MMP inhibitor is unlikely to have the same effect as a MMP-3 inhibitor, or a p38-mitogen activated protein kinase (MAPK) inhibitor the same as a $\gamma$-p38-MAPK inhibitor.

Finally, although the enthusiasm of a pharmaceutical company that is close to launching a potential blockbuster is understandable, a critical evaluation of the drug's best benefit/risk ratio must be carefully conducted. It should be checked before and after launch, because unexpected side effects may occur once the drug is used in the real world. Well designed post-marketing surveillance is mandatory.

Although developing a disease-modifying anti-osteoarthritic drug that delays joint breakdown in OA appears Utopian, like the quest for the Holy Grail, it is worthwhile. The dramatic ageing of the population and the increase in obesity in developed countries will prove extremely costly both economically and in terms of quality of life. We should keep in mind that behind all of the revolutionary drugs presently on the market is a long history of failure. If we continue to develop and test hypotheses, then one day we will find the Holy Grail!

\section{Competing interests}

$\mathrm{FB}$ is, or has been a consultant for the following companies in the past 5 years: Pfizer, Novartis, UCB, Nicox, CombinatorX, Expanscience, AstraZeneca, Takeda, Negma. FB is a member of the data safety monitoring board for Nicox.

\section{References}

1. Krzeski P, Buckland-Wright C, Balint G, Cline GA, Stoner K, Lyon R, Beary J, Aronstein WS, Spector TD: Development of musculoskeletal toxicity without clear benefit after administration of PG 116800, a matrix metalloproteinase inhibitor, to patients with knee osteoarthritis: a randomized, 12 month, double-blind, placebo-controlled study. Arthritis Res Ther 2007, 9:R109.
2. Bingham $\mathrm{CO}$, Buckland-Wright JC, Garnero $\mathrm{P}$, Cohen SB, Dougados M, Adami S, Clauw DJ, Spector TD, Pelletier JP, Raynauld JP, et al.: Risedronate decreases biochemical markers of cartilage degradation but does not decrease symptoms or slow radiographic progression in patients with medial compartment osteoarthritis of the knee: results of the two-year multinational knee osteoarthritis structural arthritis study. Arthritis Rheum 2006, 54:3494-3507.

3. Chung ES, Packer M, Lo KH, Fasanmade AA, Willerson JT; AntiTNF Therapy Against Congestive Heart Failure Investigators: Randomized, double-blind, placebo-controlled, pilot trial of infliximab, a chimeric monoclonal antibody to tumor necrosis factor-alpha, in patients with moderate-to-severe heart failure: results of the anti-TNF Therapy Against Congestive Heart Failure (ATTACH) trial. Circulation 2003, 107:3133-3140.

4. Page-McCaw A, Ewald AJ, Werb Z: Matrix metalloproteinases and the regulation of tissue remodelling. Nat Rev Mol Cell Biol 2007, 8:221-233.

5. Pitluk Z, Khalil I: Achieving confidence in mechanism for drug discovery and development. Drug Discov Today 2007, 12:924930 . 